\title{
The Simultaneous Block Triangular Decoupling, Disturbance Rejection and Pole Assignment Problem for Linear Multivariable Systems ${ }^{\dagger}$
}

\author{
Naohisa OTSUKA* and Hiroshi INABA**
}

\begin{abstract}
This paper studies the problem of simultaneously achieving block triangular decoupling, disturbance rejection and pole assignment by state feedback for linear multivariable systems in the so-called geometric approach, and presents necessary and sufficient conditions for this simultaneous problem to be solvable.
\end{abstract}

Key words : block triangular decoupling, disturbance rejection, pole assignment, geometric approach

\section{Introduction}

The block triangular decoupling problem for linear multivariable systems was first studied in the socalled geometric approach by Morse and Wonham ${ }^{2}$, and necessary and sufficient conditions for the problem to be solvable were obtained. Furthermore, they investigated the problem of simultaneous block triangular decoupling and pole assignment, and gave necessary and sufficient conditions for its solvability. On the other hand, the problem of simultaneous disturbance rejection and pole assignment was first discussed in the same approach by Chang and Rhodes ${ }^{1)}$, and necessary and sufficient conditions for its solvability were obtained. Recently, the problem of simultaneous block triangular decoupling and disturbance rejection was studied in the geometric approach by Otsuka ${ }^{3)}$ and necessary and sufficient conditions for its solvability were obtained.

The objective of this paper is to study the problem of simultaneous block triangular decoupling, disturbance rejection and pole assignment by state feedback for linear multivariable systems in the geomet-

$\dagger$ Presented at the 14th Dynamical System Theory Symposium $(1991 \cdot 12)$

* Institute of Information Sciences and Electronics, University of Tsukuba, Tsukuba

** College of Science and Engineering, Tokyo Denki University, Hatoyama-Machi, Hiki-Gun, Saitama (Received July 23, 1991)

(Revised January 22, 1992) ric approach, and to give necessary and sufficient conditions for this simultaneous problem to be solvable. Section 2 gives preliminaries and the problem formulation. In Section 3, the main results are given. Some concluding remarks are given in Section 4.

\section{Preliminaries and the Problem Formulation.}

We consider the following time invariant linear system :

(2.1) $\Sigma: \frac{d}{d x} x(t)=A x(t)+B u(t), x(0)=x_{0} \in X$, where $x(t) \in X:=\boldsymbol{R}^{n}$ is the state vector, $u(t) \in U$ : $=\boldsymbol{R}^{m}$ is the input vector, $A$ and $B$ are matrices with appropriate sizes. First, we give some definitions and notations which will be used throughout the following discussion.

Let $\Gamma \subset X$ be a subspace. Then, $\Gamma$ is said to be ( $A$, $B$ )-invariant if there exists an $F \in \boldsymbol{R}^{m \times n}$ such that ( $A$ $+B F) \Gamma \subset \Gamma$. We denote by $\mathbf{F}(A, B ; \Gamma)$ the set of all those $F$ satisfying the above inclusion. Further, we introduce the following notations: $\langle A \mid \Gamma\rangle:=\Gamma+A \Gamma$ $+\cdots+A^{n-1} \Gamma, \operatorname{Im} A:=$ the image of $A, \sigma(A):=$ the set of eigenvalues of $A$, and $\left.A\right|_{\Gamma}:=$ the restriction $A$ to $\Gamma$ as a linear map. The subspace $\langle A \mid \operatorname{Im} B\rangle$ is called the controllable subspace for System (2.1), and System (2.1) is controllable if and only if $\langle A \mid \operatorname{Im} B\rangle=X$. A subspace $\Phi \subset X$ is called a controllability subspace for (2.1) if there exist matrices $F \in \boldsymbol{R}^{m \times n}$ and $G \in \boldsymbol{R}^{m \times m}$ 
so as to satisfy $\Phi=\langle A+B F \mid \operatorname{Im} B G\rangle$. Finally, we introduce two families of subspaces as follows:

$\mathbf{V}(A, B ; \Gamma):=\{V \subset \Gamma \mid V$ is an $(A, B)$ -invariant subspace\},

and

$\mathrm{C}(A, B ; \Gamma):=\{V \subset \Gamma \mid V$ is a controllability subspace for (2.1)\}.

Now, we quote the following two lemmas.

(2.2) Lemma ${ }^{6}$. If $\Phi$ is a controllability subspace for (2.1), then

$\Phi=\langle A+B F \mid \operatorname{Im} B \cap \Phi\rangle$ for all $F \in \mathbf{F}(A, B ; \Phi)$.

(2. 3) Lemma ${ }^{6)} . \mathbf{V}(A, B ; \Gamma)$ and $\mathbf{C}(A, B ; \Gamma)$ have their supremal elements $V^{*}(\Gamma)$ and $\Phi^{*}(\Gamma)$, respectively.

The following lemma plays an important role to prove one of the main theorems in Section 3.

(2.4) Lemma ${ }^{7}$. Let System (2.1) be controllable and let $V \in \mathbf{V}(A, B ; X)$ with $\operatorname{dim}(V)=\alpha$. If $F_{0}$ is in $\mathbf{F}(A, B ; V)$ and $\Lambda$ is a symmetric set of $n-\alpha$ complex numbers, there exists a matrix $F \in \boldsymbol{R}^{m \times n}$ such that

$$
\begin{aligned}
\left.F\right|_{V} & =\left.F_{0}\right|_{V} \text { and } \sigma(A+B F) \\
& =\sigma\left[\left.\left(A+B F_{0}\right)\right|_{V}\right] \cup \Lambda
\end{aligned}
$$

where $\left.F\right|_{V}$ indicates the restriction of $F$ onto $V$. $\square$

Next, consider System (2.1) with $k(\geqq 2)$ blockoutputs $z_{1}(t), \cdots, z_{k}(t)$ and unknown disturbance $\xi(t)$ given by

$$
\left\{\begin{array}{l}
\frac{d}{d x} x(t)=A x(t)+B u(t)+K \xi(t), \\
x(0)=x_{0} \in X \\
z_{i}(t)=D_{i} x(t) \quad(i=1, \cdots, k)
\end{array}\right.
$$

where $A$ and $B$ are as in (2.1), $K \in \boldsymbol{R}^{n \times q}$, and $D_{i}(\neq 0)$ $\in \boldsymbol{R}^{p_{i} \times n} \quad$ (For simplicity $\left.D:=\left[D_{1}^{\mathrm{T}} \cdots D_{k}^{\mathrm{T}}\right]^{\mathrm{T}}\right)$. For this system, apply a state feedback of the form

(2.6) $u(t)=F x(t)+\sum_{i=1}^{k} G_{i} v_{i}(t)$,

where $F \in \boldsymbol{R}^{m \times n}, G_{i} \in \boldsymbol{R}^{m \times m}$ and $v_{i}(t)$ are the new external inputs. Then, we have the following closed loop system :

$$
\left\{\begin{array}{c}
\frac{d}{d t} x(t)=(A+B F) x(t)+\sum_{i=1}^{k} B G_{i} v_{i}(t) \\
\quad+K \xi(t), x(0)=x_{0} \in X \\
z_{i}(t)=D_{i} x(t)(i=1, \cdots, k) .
\end{array}\right.
$$

Our simultaneous block triangular decoupling, disturbance rejection and pole assignment problem is to find (if possible) a state feedback of the form (2.6), i.e., matrices $F$ and $G_{i}(i=1, \cdots, k)$, such that the corresponding closed loop system (2.7) achieves simultaneously the following three requirements: (i ) the block-outputs $z_{1}(t), \cdots, z_{k}(t)$ are triangularly decoupled through the corresponding inputs $v_{1}(t), \cdots$, $v_{k}(t)$, i.e., each $v_{i}(t)$ controls the corresponding block-output $z_{i}(t)$, but does not affect the previous $z_{1}(t), \cdots, z_{i-1}(t)$, (ii ) disturbance $\xi(t)$ is rejected from all block-outputs $z_{i}(t)$, i.e., all $z_{i}(t)$ are independent of $\xi(t)$, and (iii) the poles of (2.7) coincide with an arbitrarily given symmetric set of $n$ complex numbers.

The requirement (ii) above has been shown to be equivalent to existence of an $(A, B)$-invariant subspace $V \subset X$ such that $\operatorname{Im} K \subset V \subset \operatorname{Ker} D^{4)}$. Noticing that the controllability subspace $\Phi_{i}$ generated by the input $v_{i}$ is given by

(2.8) $\Phi_{i}=\left\langle A+B F \mid \operatorname{Im} B G_{i}\right\rangle \quad(i=1, \cdots, k)$, it is not difficult to see that our problem above can be formulated in the following compact form ${ }^{3), 5)}$.

(2.9) Simultaneous Block Triangular Decoupling, Disturbance Rejection and Pole Assignment Problem. Given $A, B, K, D_{i}(i=1, \cdots, k)$ of $(2.5)$ and a symmetric set $\Lambda=\left\{\lambda_{1}, \cdots, \lambda_{n}\right\} \subset C$ of $n$ complex numbers, find (if possible) controllability subspaces $\Phi_{1}, \cdots$, $\Phi_{k}$ for (2.5) and an $(A, B)$-invariant subspace $V \subset X$ such that

( i ) $\Phi_{i}+\operatorname{Ker} D_{i}=X \quad(i=1, \cdots, k)$,

(ii ) $\Phi_{i} \subset \bigcap_{j=1}^{i-1} \operatorname{Ker} D_{j}=: \Gamma_{i}(i=2, \cdots, k), \Gamma_{1}:=X$,

(iii) $\operatorname{Im} K \subset V \subset \operatorname{Ker} D=: \Gamma$,

(iv) $\bigcap_{i=1}^{k} \mathbf{F}\left(A, B ; \Phi_{i}\right) \cap \mathbf{F}(A, B ; V) \neq \varnothing$ and

(v) there exists

$$
F \in \bigcap_{i=1}^{k} \mathbf{F}\left(A, B ; \Phi_{i}\right) \cap \mathbf{F}(A, B ; V)
$$

such that $\sigma(A+B F)=\Lambda$.

We call a set $\left\{\Phi_{1}, \cdots, \Phi_{k}, V\right\}$ of subspaces satisfying the above conditions ( $\mathrm{i})-(\mathrm{v})$ a solution of Problem (2.9). The problem of finding controllability subspaces $\Phi_{1}, \cdots, \Phi_{k}$ for (2.5) and an $(A, B)$-invariant subspace $V \subset X$ satisfying the conditions (i ) - (iv) is called the simultaneous block triangular decoupling and disturbance rejection problem. Further, the problem of finding an $(A, B)$-invariant subspace $V \subset X$ satisfying condition (iii) and the conditions (iv), (v) 
replaced with $\mathbf{F}(A, B ; V) \neq \varnothing$ and ${ }^{\exists} F \in \mathbf{F}(A, B$; $V)$ such that $\sigma(A+B F)=\Lambda$ is called the simultaneous disturbance rejection and pole assignment problem. Moreover, the problem of finding controllability subspaces $\Phi_{1}, \cdots, \Phi_{k}$ for (2.5) satisfying the conditions (i ) and (ii), and the conditions (iv), (v) replaced with $\bigcap_{i=1}^{k} \mathbf{F}\left(A, B ; \Phi_{i}\right) \neq \varnothing$ and ${ }^{\exists} F \in \bigcap_{i=1}^{k} \mathbf{F}\left(A, B ; \Phi_{i}\right)$ such that $\sigma(A+B F)=\Lambda$ is called the simultaneous block triangular decoupling and pole assignment problem.

Now, let us use the following notations: $V^{*}:=\mathrm{a}$ supremal element of $\mathbf{V}(A, B ; \Gamma), \Phi^{*}:=$ a supremal element of $\mathbf{C}(A, B ; \Gamma), V_{i}^{*}:=$ a supremal element of $\mathbf{V}\left(A, B ; \Gamma_{i}\right)(i=1, \cdots, k)$ and $\Phi_{i}^{*}:=$ a supremal element of $\mathbf{C}\left(A, B ; \Gamma_{i}\right)(i=1, \cdots, k)$. Then, the following theorems have been shown.

(2.10) Theorem ${ }^{1,6)}$. The disturbance rejection problem is solvable if and only if $\operatorname{Im} K \subset V^{*}$. Further, the simultaneous disturbance rejection and pole assignment problem is solvable if and only if $\operatorname{Im} K$ $\subset \Phi^{*}$ and $X=\langle A \mid \operatorname{Im} B\rangle$.

(2.11) Theorem ${ }^{2)}$. The block triangular decoupling problem is solvable if and only if $\Phi_{i}^{*}+\operatorname{Ker} D_{i}$ $=X(i=1, \cdots, k)$. Further, the simultaneous block triangular decoupling and pole assignment problem is solvable if and only if $\Phi_{i}^{*}+\operatorname{Ker} D_{i}=X(i=1, \cdots, k)$ and $X=\langle A \mid \operatorname{Im} B\rangle$.

Recently, the following theorem has been shown.

(2.12) Theorem ${ }^{3)}$. The simultaneous block triangular decoupling and disturbance rejection problem is solvable if and only if

(i ) $\Phi_{i}^{*}+\operatorname{Ker} D_{i}=X(i=1, \cdots, k)$, and

(ii) $\operatorname{Im} K \subset V^{*}$.

And in this case $\left\{\Phi_{1}^{*}, \cdots, \Phi_{k}^{*}, V^{*}\right\}$ is a solution of the problem.

Using Theorems (2.10), (2.11) and (2.12), the following corollary can be easily obtained.

(2.13) Corollary. The simultaneous block triangular decoupling and disturbance rejection problem is solvable if and only if

(i) the block triangular decoupling problem is solvable,

(ii) the disturbance rejection problem is solvable.

\section{The Main Results}

The following theorem is our main result.

(3.1) Theorem. Simultaneous block triangular decoupling, disturbance rejection and pole assignment problem (2.9) is solvable if and only if

( i ) $\Phi_{i}^{*}+\operatorname{Ker} D_{i}=X(i=1, \cdots, k)$,

(ii) $\operatorname{Im} K \subset \Phi^{*}$, and

(iii) $X=\langle A \mid \operatorname{Im} B\rangle$ (i. e., The system is controllable), and in this case $\left\{\Phi_{1}^{*}, \cdots, \Phi_{k}^{*}, \Phi^{*}\right\}$ is a solution of Problem (2.9).

Proof. (Necessity) The proof of necessity easily follows from Theorems (2.10) and (2.11).

(Sufficiency) It suffices to show that under the conditions (i )-(iii) the set $\left\{\Phi_{1}^{*}, \cdots, \Phi_{k}^{*}, \Phi^{*}\right\}$ is a solution of Problem (2.9).

First, it is clear that the set satisfies the condition (i ) of (2.9). Further, since each $\Phi_{i}^{*}$ belongs to $\mathbf{C}(A$, $\left.B ; \Gamma_{i}\right)$, we have

$$
\begin{aligned}
& \Phi_{i}^{*} \subset \Gamma_{i}:=\bigcap_{j=1}^{i-1} \operatorname{Ker} D_{j}(i=2, \cdots, k), \\
& \Phi_{1}^{*} \subset \Gamma_{1}:=X,
\end{aligned}
$$

which shows that the condition (ii) of (2.9) is satisfied. The condition (iii) of (2.9) is also satisfied because

$$
\operatorname{Im} K \subset \Phi^{*} \subset \Gamma:=\operatorname{Ker} D .
$$

What remains to be shown is that the set $\left\{\Phi_{1}^{*}, \cdots\right.$, $\left.\Phi_{k}^{*}, \Phi^{*}\right\}$ satisfies the conditions (iv), ( v ) of (2.9), i.e., that for an arbitrarily given symmetric set $\Lambda:=\left\{\lambda_{1}\right.$, $\left.\cdots, \lambda_{n}\right\}$ of $n$ complex numbers there exists an $F$ $\in \boldsymbol{R}^{m \times n}$ satisfying

$$
\begin{gathered}
\mathbf{F} \in \bigcap_{i=1}^{k} \mathbf{F}\left(A, B ; \Phi_{i}^{*}\right) \cap \boldsymbol{F}\left(A, B ; \Phi^{*}\right) \neq \varnothing \\
\text { and } \sigma(A+B F)=\Lambda .
\end{gathered}
$$

To show this, first set $\Phi_{k+1}^{*}:=\Phi^{*}$, and note that from the supremarity of $\Phi_{i}^{*}(i=1, \cdots, k+1)$ and the inclusions $\Gamma \subset \Gamma_{k} \subset \cdots \subset \Gamma_{1}=X$ it follows that

$$
\Phi_{k+1}^{*} \subset \Phi_{k}^{*} \subset \cdots \subset \Phi_{1}^{*}=\langle A \mid \operatorname{Im} B\rangle=X .
$$

Next, define subspaces $X_{1}, \cdots, X_{k}, X_{k+1}$ of $X$ by the following relations :

$$
X_{k+1}:=\Phi_{k+1}^{*}, \Phi_{i}^{*}=X_{i} \oplus \Phi_{i+1}^{*}(i=1, \cdots, k)
$$

where $\oplus$ means a direct sum of subspaces. Then, it is not difficult to see that $X_{1}, \cdots, X_{k+1}$ are linearly independent, and hence noticing $\Phi_{1}^{*}=X$ it follows that

$$
X=X_{1} \oplus \cdots \oplus X_{k} \oplus X_{k+1} .
$$

Now, introduce the projection matrix $P_{i}$ of $X$ onto $X_{i}$ 
along $\sum_{j=1, j \neq i}^{k+1} X_{j}$. Further, let $F_{i} \in \mathbf{F}\left(A, B ; \Phi_{i}^{*}\right)(i=1$, $\cdots, k+1)$, and define a matrix $F \in \boldsymbol{R}^{m \times n}$ by

$$
F:=\sum_{i=1}^{k+1} F_{i} P_{i}
$$

which is well-defined by virtue of (4).

Then, we claim that

$$
F \in \bigcap_{i=1}^{k+1} \mathbf{F}\left(A, B ; \Phi_{i}^{*}\right)
$$

which will be verified with the following two steps (a) and (b).

(a) Since $X_{k+1}=\Phi_{k+1}^{*}=\Phi^{*}$ it follows from (5) that

$$
(A+B F) \Phi_{k+1}^{*}=\left(A+B F_{k+1}\right) \Phi_{k+1}^{*} \subset \Phi_{k+1}^{*},
$$

proving $F \in \mathbf{F}\left(A, B ; \Phi_{k+1}^{*}\right)$.

(b) Let $1<q \leqq k+1$ and suppose that the inclusion

$$
(A+B F) \Phi_{q}^{*} \subset \Phi_{q}^{*}
$$

holds. Then, by ( 2 ), ( 3 ), ( 5 ) and ( 7 ), we obtain

$$
\begin{aligned}
(A+B F) \Phi_{q-1}^{*} & =\left(A+B F_{q-1}\right) X_{q-1}+(A+B F) \Phi_{q}^{*} \\
& \subset \Phi_{q-1}^{*}+\Phi_{q}^{*}=\Phi_{q-1}^{*} .
\end{aligned}
$$

Consequently, our claim ( 6 ) follows from (a) and (b).

Next, we set

$$
W_{i}:=\left\{u \in \boldsymbol{R}^{m} \mid B u \in \Phi_{i}^{*}\right\} \quad(i=1, \cdots, k+1) .
$$

Then, ( 2 ) implies that

$$
W_{k+1} \subset W_{k} \subset \cdots \subset W_{1}=\boldsymbol{R}^{m} .
$$

Now, introduce subspaces $U_{2}, \cdots, U_{k+1}$ of $\boldsymbol{R}^{m}$ by the relations $W_{i}=U_{i+1} \oplus W_{i+1}(i=1, \cdots, k)$. Then, noticing the relation $\boldsymbol{R}^{m}=W_{1}=U_{2} \oplus U_{3} \oplus \cdots \oplus U_{k+1} \oplus W_{k+1}$, we can introduce the projection matrix $G_{i}$ of $\boldsymbol{R}^{m}$ onto $W_{i}$ along $U_{2} \oplus U_{3} \oplus \cdots \oplus U_{i}(i=2, \cdots, k+1)$ and $G_{1}:=I$. Then it is clear that $G_{1} G_{2} \cdots G_{i}=G_{i}$ and $\operatorname{Im} B \cap \Phi_{i}^{*}$ $=\operatorname{Im} B G_{i}(i=1, \cdots, k+1)$. Thus, it follows from ( 6 ) and Lemma (2.2) that

$$
\Phi_{i}^{*}=\left\langle A_{F} \mid \operatorname{Im} B G_{i}\right\rangle \quad(i=1, \cdots, k+1)
$$

where $A_{F}:=A+B F$.

We first note that by virtue of ( 8$)$ the pair $\left(A_{F}\right.$, $B G_{k+1}$ ) can be interpreted as a controllable system with its state space $\Phi_{k+1}^{*}$, and hence that there exists a matrix $F_{k+1}^{\dagger} \in \boldsymbol{R}^{m \times n}$ such that

$$
\sigma\left[\left.\left(A_{F}+B G_{k+1} F_{k+1}^{\dagger}\right)\right|_{\Phi_{k+1}^{*}}\right]=\left\{\lambda_{1}, \cdots, \lambda_{\sigma_{k+1}}\right\}=: \Lambda_{k+1}
$$

where $\sigma_{k+1}$ is the dimension of $\Phi_{k+1}^{*}$. Similarly, interpretting the pair $\left(A_{F}, B G_{k}\right)$ to be a controllable system with its state space $\Phi_{k}^{*}$, we can consider $\Phi_{k+1}^{*}$ to be an $\left(A_{F}, B G_{k}\right)$-invariant subspace of $\Phi_{k}^{*}$, i.e., $\Phi_{k+1}^{*}$ $\in V\left(A_{F}, B G_{k} ; \Phi_{k}^{*}\right)$. Furthermore, we also note that $F_{k+1}^{*}:=G_{k+1} F_{k+1}^{\dagger} \in \mathbf{F}\left(A_{F}, B G_{k} ; \Phi_{k+1}^{*}\right)$.

Now, it can be seen from Lemma (2.4) and (9) that there exists a matrix $F_{k}^{\dagger} \in \boldsymbol{R}^{m \times n}$ such that

$$
\left.F_{k}^{\dagger}\right|_{\Phi_{k+1}^{*}}=\left.F_{k+1}^{*}\right|_{\Phi_{k+1}^{*}}
$$

and

$$
\begin{aligned}
& \left.\sigma\left(A_{F}+\left.B G_{k} F_{k}^{\dagger}\right|_{\Phi_{k}^{*}}\right)=\left.\sigma\left(A_{F}+B G_{k} F_{k+1}^{*}\right)\right|_{\Phi_{k+1}^{*}}\right] \cup \Lambda_{\bar{k}}^{-} \\
& \left.\quad=\left.\sigma\left(A_{F}+B G_{k+1} F_{k+1}^{+}\right)\right|_{\Phi_{k+1}^{*}}\right) \cup \Lambda_{k}^{-} \\
& \quad=\Lambda_{k+1} \cup \Lambda_{k}^{-} \\
& \quad=\left\{\lambda_{1}, \cdots, \lambda_{\alpha_{k+1}}, \lambda_{\alpha_{k+1}+1}, \cdots, \lambda_{\alpha_{k}}\right\}=: \Lambda_{k}
\end{aligned}
$$

where $\Lambda_{k}^{-}:=\left\{\lambda_{\alpha_{k+1}+1}, \cdots, \lambda_{\alpha_{k}}\right\}$ and $\alpha_{k}$ is the dimension of $\Phi_{k}^{*}$. Again, interpreting $\Phi_{k}^{*}$ to be an $\left(A_{F}, B G_{k-1}\right)$ invariant subspace of $\Phi_{k-1}^{*}$, we have $F_{k}^{*}:=G_{k} F_{k}^{+}$ $\in \mathbf{F}\left(A_{F}, B G_{k-1} ; \Phi_{k}^{*}\right)$.

Thus, we have extended the mapping $F_{k+1}^{\dagger}$ to $F_{k}^{\dagger}$ so as to satisfy (10) and (11) with $F_{k+1}^{*}=G_{k+1} F_{k+1}^{\dagger}$ $\in \mathbf{F}\left(A_{F}, B G_{k} ; \Phi_{k+1}^{*}\right)$ and $F_{k}^{*}=G_{k} F_{k}^{\dagger} \in \mathbf{F}\left(A_{F}, B G_{k-1}\right.$; $\left.\Phi_{R}^{*}\right)$. Repeating this extension we finally obtain a matrix $F_{1}^{\dagger} \in \boldsymbol{R}^{m \times n}$ such that

$$
\begin{aligned}
& \left.F_{1}^{\dagger}\right|_{\Phi_{2}^{*}}=\left.F_{2}^{*}\right|_{\Phi_{2}^{*}}, \\
& \sigma\left(A_{F}+B G_{1} F_{1}^{\dagger}\right)=\Lambda_{2} \cup \Lambda_{1}^{-}=\left\{\lambda_{1}, \cdots, \lambda_{n}\right\}=\Lambda .
\end{aligned}
$$

Furthermore, $F_{1}^{*}=G_{1} F_{1}^{+}=F_{1}^{+} \in \mathbf{F}\left(A_{F}, B ; \Phi_{1}^{*}\right)$ since $G_{1}=I$. Noticing that $A_{F}+B G_{1} F_{1}^{+}=A+B\left(F+F_{1}^{\dagger}\right)$ and defining $F^{*}:=F+F_{1}^{\dagger}$, we see from (13) that $\sigma(A$ $\left.+B F^{*}\right)=\Lambda$. Moreover, it can be shown from the construction of $F^{\dagger}$ above that

$$
F^{*} \in \bigcap_{i=1}^{k+1} \mathbf{F}\left(A, B ; \Phi_{i}^{*}\right) \neq \varnothing .
$$

In fact, using ( 6 ) with the relations $G_{1} G_{2} \cdots G_{i}=G_{i}$ and $\left.F_{1}^{\dagger}\right|_{\Phi_{i}^{*}}=\left.G_{i} F_{i}^{\dagger}\right|_{\Phi_{i}^{*}}$, it follows that

$$
\begin{aligned}
\left(A+B F^{*}\right) \Phi_{i}^{*} & =(A+B F) \Phi_{i}^{*}+B F_{1}^{\dagger} \Phi_{i}^{*} \\
& =(A+B F) \Phi_{i}^{*}+B G_{i} F_{i}^{\dagger} \Phi_{i}^{*} \\
& \subset \Phi_{i}^{*} \quad(i=1, \cdots, k+1)
\end{aligned}
$$

showing that (14) holds. Hence, (13) and (14) show that $F^{*}$ satisfies $(1)$. Therefore, $\left\{\Phi_{1}^{*}, \cdots, \Phi_{k}^{*}, \Phi^{*}\right\}$ is a solution of Problem (2.9). This completes the proof of Theorem (3.1).

Using Theorems (2.10) and (2.11), the following corollary can be easily obtained.

(3.2) Corollary. Simultaneous block triangular decoupling, disturbance Rejection and pole assignment problem (2.9) is solvable if and only if

(i) the block triangular decoupling problem is solvable. 
(ii) the simultaneous disturbance rejection and pole assignment problem is solvable.

\section{Conclusions}

This paper considered the problem of simultaneously achieving block triangular decoupling, disturbance rejection and pole assignment by state feedback, and presented necessary and sufficient conditions for this problem to be solvable.

\section{References}

1) M. F. Chang and I. B. Rhodes: Disturbance Localization in Linear Systems with Simultaneous Decoupling, Pole Assignment, or Stabilization, IEEE Trans. Automatic Control, AC-20-4, 518/523 (1975)

2) A.S. Morse and W. M. Wonham: Triangular Decoupling of Linear Multivariable Systems, IEEE Trans. Automatic Control, AC-15-4, 447/449 (1970)

3) N. Otsuka: Simultaneous Block Triangular Decoupling and Disturbance Rejection Problem, Trans. of the SICE, 27-4, 486/488 (1991) (in Japanease)

4) E. D. Sontag: Mathematical Control Theory: Deterministic Finite Dimensional Systems, Springer, New York (1990)

5) W.M. Wonham: On Pole Assignment in Multiinput Controllable Linear Systems, IEEE Trans., AC-12-6, $660 / 665$ (1967)

6) W.M. Wonham and A.S. Morse: Decoupling and Pole Assignment in Linear Multivariable Systems : A Geometric Approach, SIAM J. on Control, 8, 1/18 (1970)

7) W.M. Wonham: Linear Multivariable Control: A Geometric Approach, Springer, New York (1979)

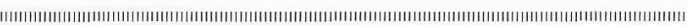

\section{Naohisa OTsuka (Member)}

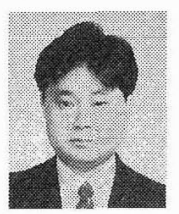

He was born in Niigata on April 29, 1960, and he received the $\mathrm{Ph}$. D. degree in mathematical sciences from Tokyo Denki University, Japan, in 1992.

From 1986 to 1991 he served as a Research Associate in the Department of Information Sciences, Tokyo Denki University. Since April 1992, he has been a Research Associate in the Institute of Information Sciences and Electronics, University of Tsukuba. His research interests include decoupling problems and infinite-dimensional systems.

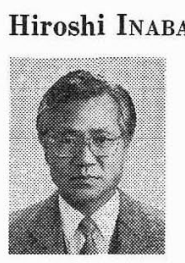

\section{(Member)}

He was born in Hokkaido on Feburary 9,1937 . He received the $\mathrm{Ph}$. D. degree in aerospace engineering from the University of Texas at Austin in 1972.

From 1974 to 1976 he served as an Assistant Professor in the Department of Aerospace Engineering, the University of Texas at Austin. In 1977 he joined as a Professor in the Department of Mathematical Sciences, Tokyo Denki University, and since 1986 he has been with the Department of Information Sciences in the same university. His main research interest is in mathematical system theory, in particular, the theories of infinite-dimensional linear systems and algebraic linear systems. 